\title{
Fibras altamente robustas de microextração em fase sólida e recobrimento via reação sol-gel
}

\author{
Eduardo Carasek', Edmar Martendal', Dilma Budziak² \\ ${ }^{1}$ Departamento de Química, Universidade Federal de Santa Catarina, \\ Cep 88040-900, Florianópolis, SC, Brasil \\ e-mail: carasek@qmc.ufsc.br \\ ${ }^{2}$ Campus Curitibanos, Universidade Federal de Santa Catarina, \\ Cep 89520-000, Curitibanos, SC, Brasil
}

\section{Resumo}

O aperfeiçoamento de técnicas de preparação de amostras baseadas em sorção e/ou o desenvolvimento de novos materiais é extremamente importante para ampliar sua aplicabilidade, eficiência e robustez. A fragilidade da sílica fundida e a necessidade de suportes robustos para microextração em fase sólida (SPME), bem como uma eficiente interação entre o recobrimento extrator e o suporte, têm levado ao desenvolvimento e aplicação de novos materiais. Neste artigo, será discutido o uso de fios metálicos na confecção de fibras altamente robustas de SPME. Em especial, será destacado o uso da liga de NiTi recoberta com $\mathrm{ZrO}_{2}$ por eletrodeposição, a qual será avaliada como suporte e sorvente extrator para SPME, bem como substrato para reações sol-gel com poli-dimetil-siloxano (PDMS) e poli-etilenoglicol (PEG).

\section{Palavras-chave}

Microextração em fase sólida; suporte metálico; Ni-Ti; cromatografia; técnicas de preparação de amostras.

\section{Unbreakable solid-phase microextraction fibers obtained by sol-gel reaction}

\section{Abstract}

Improvement of sorption based sample preparation techniques and/or the development of new materials for these same techniques are/is extremely important to extend their (its) applicability, efficiency and robustness. The fragility of fused silica and the necessity of robust supports for solid phase microextration (SPME), as well as the importance of an efficient interaction between the extractor coating and the support, have driven the development and application of new materials. In this review the use of metallic wires as a support to "unbreakable" SPME fibers will be discussed. The focus of this paper will be the use of NiTi alloy coated with $\mathrm{ZrO}_{2}$ by eletrodeposition and its evaluation as support and extracting sorvent for SPME, as well as substratum for sol-gel reactions with com polidimetilsiloxano (PDMS) and polietilenoglicol (PEG).

Keywords

Solid phase microextraction; metallic wires; $\mathrm{Ni}$-Ti; chromatography; sample preparation techniques. 


\section{Introdução}

A miniaturização tem se tornado uma das tendências dominantes em Química Analítica. Exemplos típicos da miniaturização de técnicas para a preparação de amostras incluem, principalmente, a microextração líquido-líquido ${ }^{[1]}$, discos ou cartuchos de SPE e SPE on-line ${ }^{[2,3]}$, microextração em seringa empacotada (MEPS $)^{[4-7]}$, extração com gota única $(\mathrm{SDME})^{[8]}$ e extração assistida por membrana $(\mathrm{MAE})^{[9]}$. Técnicas como essas, quando usadas em combinação com sistemas analíticos convencionais, podem resultar em análise mais rápida, levando a alta frequência analítica, menor consumo de solventes e manipulação da amostra. Além disso, essas técnicas podem manter ou até melhorar a sensibilidade, quando comparadas a técnicas convencionais. Em particular, espera-se que a redução no consumo de solventes em laboratórios analíticos contribua significativamente na redução dos custos de análise. Muitas técnicas de preparação de amostras miniaturizadas podem ser automatizadas e acopladas para análise on-line. Tal acoplamento de extração e análise favorece a total extração e transferência para o sistema analítico, resultando em alta sensibilidade e uma perda potencial do analito mais baixa. Além disso, o analista pode usar pequenos volumes de amostra ${ }^{[10]}$. Técnicas de preparação de amostras livres de solventes que são baseadas em extração por sorção vêm ganhando a atenção de pesquisadores. Dentre essas técnicas, está incluída principalmente a microextração em fase sólida (SPME) $)^{[11]}$, a qual representa um importante avanço na eficiência de extração de vários poluentes orgânicos em nível de traço ${ }^{[12]}$ de matrizes líquidas ${ }^{[13]}$, sólidas ${ }^{[14]}$ e gasosas ${ }^{[15]}$. A SPME também é usada para amostragem de compostos voláteis e semi-voláteis de amostras complexas, como lodo ${ }^{[16]}$. Adicionalmente, a
SPME também tem sido aplicada com sucesso como um dispositivo para amostragem de compostos orgânicos voláteis de amostras biológicas $^{[17-19]}$ e gêneros alimentícios ${ }^{[20-22]}$ em análise cromatográfica. O dispositivo básico de SPME consiste em um suporte (mais comum o uso de sílica fundida), com cerca de $100 \mathrm{~mm}$ de comprimento, $0,110 \mathrm{~mm}$ de diâmetro, sendo que $10 \mathrm{~mm}$ de sua extremidade são recobertos com uma fase estacionária (fase extratora). Materiais poliméricos termicamente estáveis que permitem uma difusão rápida do soluto são comumente usados como fases estacionárias ${ }^{[10]}$.

Primeiramente, somente os recobrimentos de poli(dimetilsiloxano) (PDMS) e poliacrilato (PA) eram comercializados. Mais tarde, recobrimentos com fases mistas, como PDMS-divinilbenzeno (DVB), Carboxen-PDMS, Carbowax-DVB, também foram utilizados ${ }^{[23]}$. Recentemente muitos novos tipos de fibras, como as partículas de sílica ligadas com C8, C18 $8^{[24]}$, grafite ${ }^{[25]}$, carvão ativado $^{[26]}$, alumínio anodizado ${ }^{[27]}$, polipirrol ${ }^{[28,29]}$, polianilina $^{[30-32]}$, fibra recoberta com a impressão molecular do polímero (MIP) $)^{[3,34]}$, cloreto de cobre $(\mathrm{I})^{[35]}$, entre outras, têm sido preparados.

Entretanto, muitas dessas fibras são preparadas por mera deposição física de um recobrimento polimérico sobre a superfície de sílica fundida. A ausência de uma interação adequada entre a fase estacionária e a superfície da sílica fundida pode ser responsável pela baixa estabilidade química e térmica ${ }^{[10,23,36,37]}$. É evidente que futuros avanços na tecnologia de SPME devem depender grandemente do avanço científico, levando ao desenvolvimento de tecnologias mais eficientes para a criação de fases extratoras mais seletivas e a imobilização química dessas, como filmes de elevada estabilidade operacional (temperatura, solvente etc.). A química de sol-gel oferece um simples e conveniente caminho para a síntese de materiais avançados e a aplicação deles 
como revestimentos de superfícies. A química de sol-gel pode fornecer a incorporação eficiente de componentes orgânicos em estruturas poliméricas inorgânicas sob circunstâncias térmicas extremamente suaves ${ }^{[10]}$. Outro avanço na tecnologia de SPME aponta para a substituição do frágil suporte de sílica fundida por fios metálicos que possuem boa estabilidade mecânica, tornando a SPME uma técnica de preparação de amostra mais robusta para análise de rotina.

\section{Tecnologia sol-gel para fibras de SPME}

O processo de sol-gel consiste na rota de síntese em que ocorre uma transição do sistema sol para um sistema gel. O termo sol é empregado para definir uma dispersão de partículas coloidais (dimensão entre 1 e 100 nm) estável em um fluido. O termo gel pode ser visto como referindo-se a um sistema formado pela estrutura rígida de partículas coloidais (gel coloidal), as quais resultam da agregação linear de partículas primárias ou de cadeias poliméricas (gel polimérico), que imobiliza a fase líquida nos seus interstícios, sendo que a gelatinização ocorre pela interação entre as longas cadeias poliméricas lineares.

O processo de sol-gel pode ser dividido em duas classes, dependendo da natureza do precursor inorgânico utilizado: a dos sais (cloretos, sulfetos, nitratos etc.) e a dos alcóxidos. A rota que envolve o uso de precursores do tipo alcóxido aparece como a mais versátil atualmente. A hidrólise de uma solução de tetra-alcóxi-silanos em um solvente orgânico, como o álcool, leva à formação de partículas com função silanol, as quais formam um sol pela polimerização via condensação, e a continuação do processo leva a um gel. Essa transformação é designada transição sol-gel. Após secagem do gel, um xerogel é formado. As reações químicas que ocorrem durante a formação do sol, do gel e do xerogel influenciam fortemente a composição e as propriedades do produto final ${ }^{[38]}$.

A Tabela 1 lista os nomes e as estruturas químicas dos principais ingredientes da solução usada para o recobrimento sol-gel. A solução sol-gel contém quantidades apropriadas do polímero de recobrimento PDMS ou PEG, um precursor alcóxi-silano (metil-trimetil-metóxi-silano, MTMOS), reagente de desativação da superfície (poli-metil-hidróxi-silano, PMHS), e um catalisador ácido (solução aquosa 95\% de ácido tri-fluor-acético, TFA).

O material sol-gel é formado como resultado da hidrólise do precursor alcóxi-silano com subsequente condensação dos produtos hidrolisados, podendo submeter-se ao rachamento e ao encolhimento durante a etapa de secagem. Tanto o encolhimento quanto as rachaduras são efeitos indesejáveis durante o processo de secagem do gel. As fraturas capilares são devidas à evaporação do solvente dos poros do gel. Tal problema se torna grandemente significativo para recobrimentos por sol-gel com espessuras maiores que 0,5-1 $\mu \mathrm{m}$. No contexto de SPME, a preservação da integridade da estrutura é muito importante para que o recobrimento sol-gel possa fornecer as propriedades materiais desejadas. Uma rede sol-gel originária de um derivado alquil de um percursor tetra-alcóxi-silano (por ex. metil-tri-metóxi-silano) possui uma maior abertura em sua estrutura, o que facilita a secagem, minimizando assim a tendência a fraturas ${ }^{[10]}$.

O processo sol-gel envolve duas etapas principais: (1) hidrólise do precursor e (2) policondensação dos produtos hidrolizados. Essas reações são catalisadas por ácidos e bases, levando à formação de uma rede polimérica. A reação do precursor metil-tri-metóxi-silano (MTMOS) pode ser representada pela Equação $1^{[10,38,39]}$. Os 
Tabela 1 Nomes, funções e estruturas químicas dos reagentes usados no processo sol-gel para recobrimento de fibras de SPME.

\begin{tabular}{|c|c|c|}
\hline Reagente & Função & Estrutura química \\
\hline $\begin{array}{l}\text { Metil-trimetóxi-silano } \\
\text { (MTMOS) }\end{array}$ & Precursor sol-gel & $\mathrm{H}_{3} \mathrm{CO}-\left.\right|_{\mathrm{OCH}_{3}} ^{\mathrm{CH}_{3}}-\mathrm{OCH}_{3}$ \\
\hline $\begin{array}{l}\text { Poli(dimetil-siloxano) com } \\
\text { grupo hidroxila terminal }\end{array}$ & $\begin{array}{l}\text { Recobrimento da } \\
\text { fase estacionária }\end{array}$ & $\mathrm{HO}-\left.\right|_{\mathrm{CH}_{3}} ^{\mathrm{CH}_{3}} \mathrm{O}-\left.\right|_{\mathrm{CH}_{3}} ^{\mathrm{CH}_{3}} \mathrm{O}-\left.\mathrm{I}_{\mathrm{n}}^{\mathrm{Si}}\right|_{\mathrm{CH}_{3}} ^{\mathrm{CH}_{3}} \mathrm{OH}$ \\
\hline Poli(etilenoglicol) & $\begin{array}{l}\text { Recobrimento da } \\
\text { fase estacionária }\end{array}$ & $\mathrm{HO}+\left.\left.\right|_{\mathrm{H}} ^{\mathrm{H}}\right|_{\mathrm{H}} ^{\mathrm{H}}-\mathrm{Si}_{\mathrm{H}} \mathrm{O}+\frac{\mathrm{m}}{\mathrm{H}} \mathrm{H}$ \\
\hline Poli(metil-hidrosiloxano) & $\begin{array}{c}\text { Reagente } \\
\text { desativante }\end{array}$ & 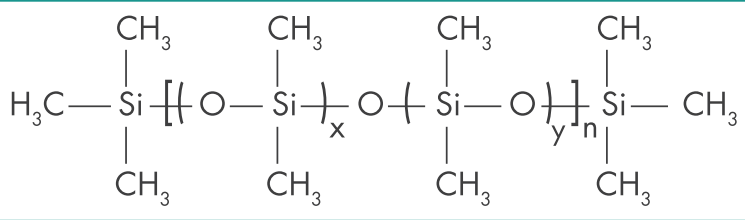 \\
\hline $\begin{array}{l}\text { Ácido tri-fluor-acético } \\
\text { (5\% de água) }\end{array}$ & Ácido catalisador & $\mathrm{CF}_{3} \mathrm{COOH}$ \\
\hline
\end{tabular}

produtos hidrolizados podem sofrer reações de policondensação e produzir uma rede polimérica tridimensional, conforme descrita pela Equação 2. Em uma solução sol-gel que utiliza poli-dimetil-siloxano, contendo um grupo hidroxila terminal como reagente de recobrimento, esse grupamento hidroxila é ativado. A reação química envolvida pode ser esquematicamente representada pela Equação $3^{[10]}$. Selecionando poli(etilenoglicol) (PEG) como polímero de recobrimento, a reação química pode ser representada pela Equação $4^{[39]}$.<smiles>CO[Si](C)(OC)O[Na]</smiles><smiles>CO[Si](C)(O)O[Si](C)(O)OI</smiles> 


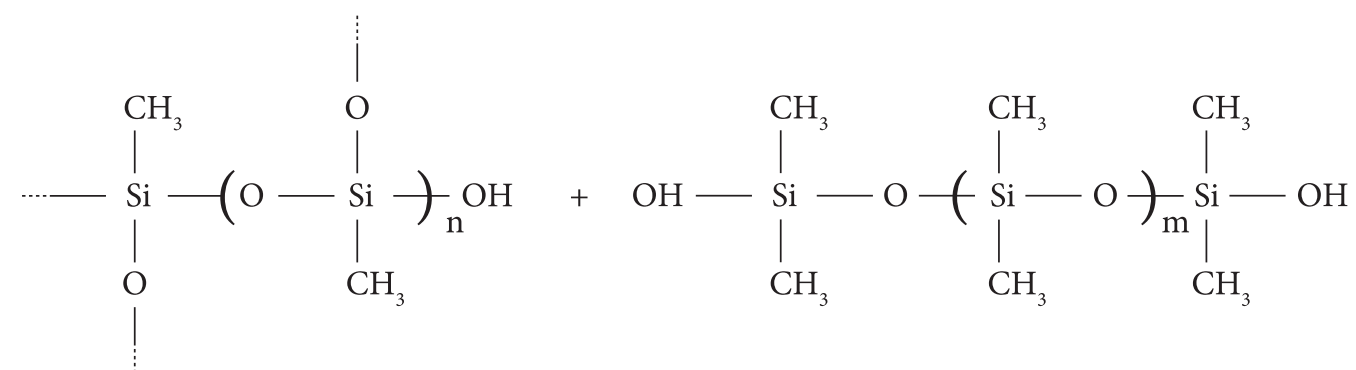<smiles>C[Si](C)(OI)O[Si](C)(C)O[Si](C)(C)O[Si](C)(C)O[Si](C)(C)OI</smiles><smiles>COI(C)[Si](C)(C)O[Si](C)(C)OI</smiles><smiles>CO[Si](C)(C)O[Si](C)(C)OCOI</smiles>

O grupo silanol da superfície dos suportes (geralmente sílica fundida) está sujeito a reações de condensação, permitindo a ligação química entre a rede polimérica e a superfície dos suportes. Dessa maneira, o recobrimento polimérico ligado à superfície do suporte é gerado pela exposição da fibra utilizada como suporte na solução sol por um tempo determinado. O recobrimento, ou filme polimérico, é quimicamente ligado à superfície do suporte, e sua espessura pode ser controlada pela variação do tempo de exposição e concentração dos componentes da solução. Recobrimentos com maiores espessuras podem ser obtidos por repetidas exposições dos suportes à solução sol-gel.

A hidrólise - primeira etapa do mecanismo do processo sol-gel - é bem conhecida. Por outro lado, as reações de condensação começam antes que as reações de hidrólise terminem, tornando o mecanismo muito complexo e envolvendo muitas reações de hidrólise e condensação simultâneas ${ }^{[38]}$. 
Historicamente, o uso da tecnologia de sol-gel é reportada desde meados de $1800^{[40]}$. Inicialmente aplicada em SPME por Chong et al. ${ }^{[10]}$ em 1997, tem sido aplicada com sucesso no preparo de diferentes fibras, como pode ser verificado por meio da Tabela 2.

\section{Uso de suportes metálicos em microextração em fase sólida}

Vários materiais vêm sendo propostos em substituição à sílica fundida, suporte tradicionalmente usado em SPME. A aplicação de fios metálicos como suportes pressupõe uma maior robustez para a técnica de SPME para análises de rotina. Diferentes materiais, tais como fios de platina ${ }^{[32,41-43]}$, fios de alumínio anodizado ${ }^{[44]}$, fios de ouro ${ }^{[31,44]}$, fibras de carbono ativado $^{[45]}$, aço inoxidável ${ }^{[30,46-48]}$, fios de titânio ${ }^{[49]}$ e fios de cobre $^{[35]}$, vêm sendo explorados em substituição ao suporte tradicional de sílica fundida. Recentemente, uma nova geração de fibras de SPME foi introduzida comercialmente pela Supelco. Essa nova fibra, denominada de fibra de metal super elástica, pode ser reutilizada em mais de 600 ciclos de extração ${ }^{[50-52]}$.

Tabela 2 Diferentes fibras para microextração em fase sólida desenvolvidas através de processo sol-gel.

\begin{tabular}{|c|c|c|c|}
\hline Recobrimento & $\begin{array}{l}\text { Espessura } \\
\text { filme }\end{array}$ & Compostos analisados & Ref. \\
\hline Poli-dimetil-siloxano (PDMS) & $10 \mu \mathrm{m}$ & HPAa, n-alcanos, álcoois e fenóis & [10] \\
\hline PDMS & $44 \mu \mathrm{m}$ & BTEXb & [53] \\
\hline Poli(etilenoglicol) (PEG) & $40 \mu \mathrm{m}$ & BTEXb, cloro e nitrofenóis, ftalatos e pesticidas & [39] \\
\hline Calix[4]areno & $60 \mu \mathrm{m}$ & Fenóis & [54] \\
\hline Éter de coroa - bisbenzo & $40 \mu \mathrm{m}$ & Pesticidas e compostos organofosforados & {$[55]$} \\
\hline PDMS/poli(vinil-álcool) (PDMS/PVA) & $5 \mu \mathrm{m}$ & Bifenilas policloradas (BPC) & {$[56]$} \\
\hline Éter de coroa aberta & $55 \mu \mathrm{m}$ & Fenóis, BTEXb e ftalatos & {$[36]$} \\
\hline Éter de coroa com hidroxila & $73 \mu \mathrm{m}$ & Fenóis & {$[37]$} \\
\hline Poli-fenil-metil-siloxano (PPMS) & $70 \mu \mathrm{m}$ & Pesticidas organoclorados & [57] \\
\hline $\begin{array}{l}\text { Tri-metoxi-silil-propil-metacrilato } \\
\text { (TMSPMA) }\end{array}$ & $70 \mu \mathrm{m}$ & Compostos voláteis da cerveja & [58] \\
\hline $\begin{array}{l}\text { Anilina-metil-trietoxi-silano/PDMS } \\
\text { (AMTEOS/PDMS) }\end{array}$ & $85 \mu \mathrm{m}$ & Compostos aromáticos & [59] \\
\hline $\begin{array}{l}\text { Butil-metacrilato/divinil-benzeno } \\
\text { (BMA/DVB) }\end{array}$ & $70 \mu \mathrm{m}$ & Compostos voláteis em vinho & [60] \\
\hline Hidroxi-fulereno & $30 \mu \mathrm{m}$ & BPC, HPAa e aminas aromáticas & {$[23]$} \\
\hline $\begin{array}{c}\text { (Tri-metoxi-silil-propil-amina)/PDMS } \\
\text { (TMSPA/OH-PDMS) }\end{array}$ & $4 \mu \mathrm{m}$ & Fenol e clorofenóis em amostras ambientais & {$[61]$} \\
\hline $\begin{array}{l}\text { Poli-metil-fenilssiloxano } \\
\text { (PMPS) }\end{array}$ & $70 \mu \mathrm{m}$ & Pesticidas em vegetais & {$[62]$} \\
\hline $\begin{array}{l}\text { Permetilado- } \beta \text {-ciclodextrina/ silicone } \\
\text { hidroxi terminal (PM- } \beta-C D / O H-T S O)\end{array}$ & $65-70 \mu \mathrm{m}$ & $\begin{array}{l}\text { Eters difenilpolibromados (PBDE) em } \\
\text { amostras de solo }\end{array}$ & {$[63]$} \\
\hline $\begin{array}{l}\text { Tetra-n-butil-ortototitanato/grafite } \\
\text { (TBOT/grafite) }\end{array}$ & $30 \mu \mathrm{m}$ & BTEXb em amostras gasosas & [64] \\
\hline
\end{tabular}

\footnotetext{
${ }^{a}$ Hidrocarbonetos policíclicos aromáticos; ${ }^{\text {b }}$ benzeno, tolveno, etilbenzeno e xileno.
} 
Esse grupo de pesquisa tem explorado as vantagens da liga aproximadamente equiatômica de NiTi, conhecida comercialmente como Nitinol $^{\oplus}$, como suporte para a confecção de fibras de SPME. Essa liga se destaca por seu efeito de memória de forma (habilidade de transformação entre fases que permite a recuperação de uma geometria previamente definida mediante imposição de gradiente de tensão e/ou temperatura) e pela superelasticidade. Tais propriedades aliadas à estável interação superficial com o ambiente externo, alta biocompatibilidade, excepcional durabilidade e resistência à corrosão são características altamente desejáveis ${ }^{[65]}$. As vantagens da liga de NiTi somadas àquelas do recobrimento com óxido de zircônio $\left(\mathrm{ZrO}_{2}\right)$, incluindo forte adesão a superfícies metálicas, excelente biocompatibilidade, alta estabilidade térmica e resistência à corrosão e ao desgaste e, principalmente, à maior disponibilidade de grupos hidroxilas sobre sua superfície sugerem que esse suporte pode oferecer uma promissora alternativa como novo suporte para a técnica de microextração em fase sólida por meio do uso da tecnologia de sol-gel. Com a ativação do suporte $\mathrm{NiTi}-\mathrm{ZrO}_{2}$ com soluções de hidróxido de sódio $1,0 \mathrm{~mol} . \mathrm{L}^{-1}$ e posterior solução de ácido clorídrico $0,5 \mathrm{~mol} . \mathrm{L}^{-1}$, supõe-se que ocorra uma exposição de grupamentos $\mathrm{Zr-OH}$ na superfície desse material, o que possibilita a aplicação do conjunto NiTi- $\mathrm{ZrO}_{2}$ como um novo suporte para reações sol-gel. Os grupos $\mathrm{Zr}-\mathrm{OH}$ na superfície do suporte podem também participar por meio de reações de condensação e assim prover ancoramento químico à cadeia polimérica na vizinhança imediata da superfície da fibra. A Figura 1 ilustra as micrografias das fibras de SPME obtidas via reação sol-gel de PDMS utilizando suporte de NiTi sem a camada de óxido de zircônio (NiTi-PDMS) e NiTi com a camada de óxido de zircônio (NiTi$\mathrm{ZrO}_{2}$-PDMS). Quando não há o filme de zircônia sobre o fio de $\mathrm{NiTi}$, o recobrimento polimérico não é uniforme e, em muitos casos, a espessura de recobrimento não é reprodutível, resultando em diferentes espessuras.

As fibras de $\mathrm{NiTi}-\mathrm{ZrO}_{2}-\mathrm{PDMS}$ e $\mathrm{NiTi}-\mathrm{ZrO}_{2}-$ PEG apresentaram excelente estabilidade térmica até $320^{\circ} \mathrm{C}$, possibilitando a aplicação de altas temperaturas de dessorção, sem perdas na eficiência de extração. A eficiência da fibra $\mathrm{NiTi}-\mathrm{ZrO}_{2}-$ PDMS com $25 \mu \mathrm{m}$ de espessura mostrou-se superior à da fibra de PDMS $30 \mu \mathrm{m}$ comercialmente disponível, apresentando excelente sensibilidade para a determinação de BTEX em água ${ }^{[66]}$ e pes-
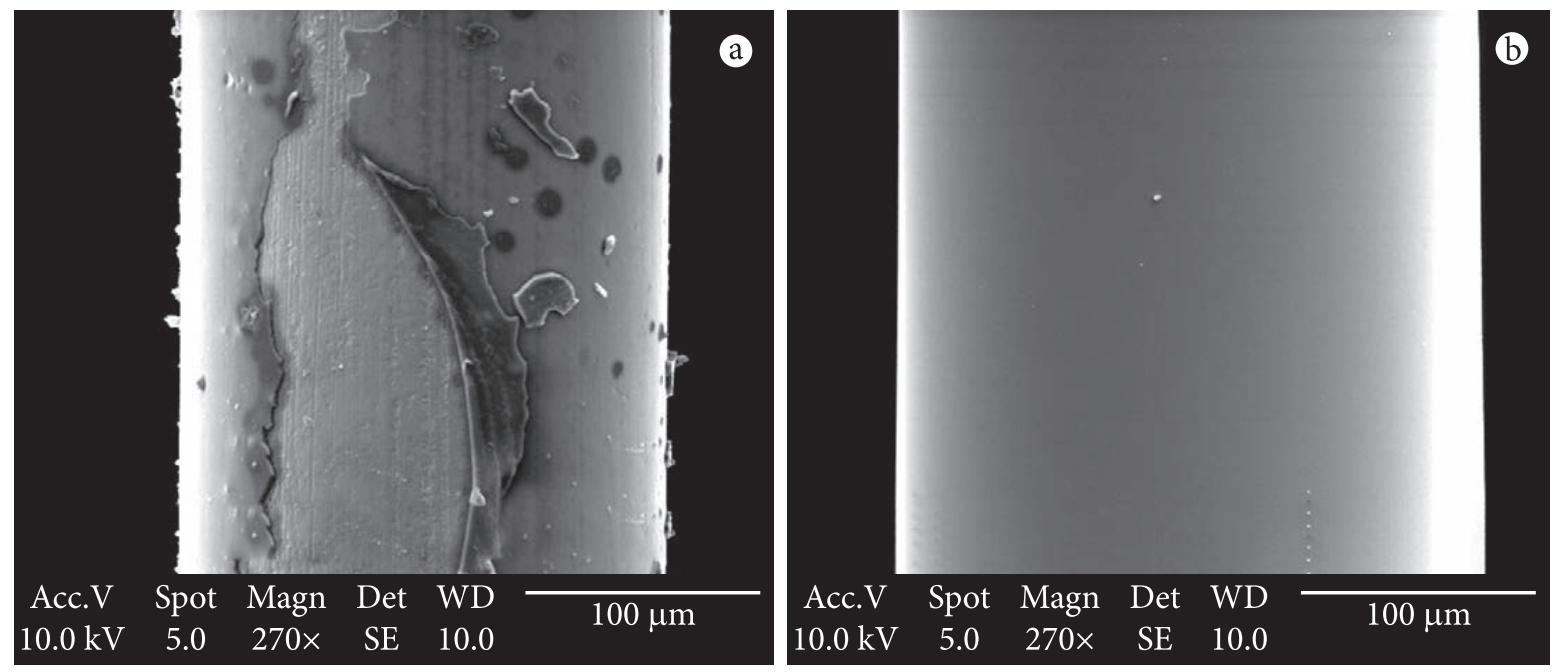

Figura 1 Micrografias eletrônicas de varredura da fibra de a) NiTi-PDMS e b) NiTi-ZrO 2 -PDMS. Ampliação $270 \times$. 
ticidas organoclorados em infusões de chá ${ }^{[67]}$. A eficiência de extração da fibra NiTi- $\mathrm{ZrO}_{2}-\mathrm{PEG}$ foi comparável à das fibras comerciais CW/DVB e $\mathrm{PA}$, apresentando excelente eficiência de extração para halofenóis e ftalatos em amostras aquosas ${ }^{[68]}$ e haloanisóis em amostras de cortiça ${ }^{[69]}$. Ambas as fibras demonstraram aplicabilidade, tanto para amostragem direta (contato direto da fibra com a solução amostra), quanto para amostragem a partir do headspace da amostra (parte gasosa), e mantiveram eficiência de extração por mais de 450 ciclos de extração.

\section{Conclusões e perspectivas}

A microextração em fase sólida com recobrimento via reação sol-gel sobre uma superfície metálica oferece um bom desempenho analítico, com boa sensibilidade e alta robustez. A alta estabilidade térmica obtida para as fibras de NiTi$\mathrm{ZrO}_{2}$-PDMS e NiTi-Z $\mathrm{rO}_{2}$-PEG sugere uma possível e eficiente ligação química entre o suporte zirconizado e o polímero de recobrimento.

\section{Agradecimentos}

Ao $\mathrm{CNPq}$, pelo suporte financeiro.

\section{Referências}

1 Leinonen A, Vuorensola K, Lepola L-M, Kuuranne T, Kotiaho T, Ketola RA. et al. Liquid-phase microextraction for sample preparation in analysis of unconjugated anabolic steroids in urine. Analytica Chimica Acta 2006; 16:559-172.

2 Lanças LM. Extração em Fase Sólida (SPE). São Carlos: RiMa; 2004.

3 Tarning J, Singtoroj T, Annerberg A, Ashton M, Bergqvist $\mathrm{Y}$, White $\mathrm{NJ}$ et al. Development and validation of an automated solid phase extraction and liquid chromatographic method for the determination of piperaquine in urine. Journal of Pharmaceutical and Biomedical Analysis 2006; 41:213-218. http://dx.doi.org/10.1016/j.jpba.2005.10.027
Abdel-Rehim M. Determination of local anaesthetics in human plasma samples using gas chromatographymass spectrometry. Journal of Chromatography $B$ 2004; 317:801-231.

5 Abdel-Rehim M, Skansen P, Vita M, Hassan Z, Blomberg L, Hassan M. Microextraction in packed syringe/liquid chromatography/electrospray tandem mass spectrometry for quantification of olomoucine in human plasma samples. Analytica Chimica Acta 2005; 539:35-39.http://dx.doi.org/10.1016/j.aca.2005.02.061

Altun Z, Abdel-Rehim M, Blomberg LG. New trends in sample preparation: on-line microextraction in packed syringe (MEPS) for LC and GC applications: Part III: Determination and validation of local anaesthetics in human plasma samples using a cation-exchange sorbent, and MEPS-LC-MS-MS. Journal of Chromatography B 2004; 813:129-135. http://dx.doi.org/10.1016/j.jchromb.2004.09.020

7 El-Beqqali A, Kussak A, Abdel-Rehim M. Fast and sensitive environmental analysis utilizing microextraction in packed syringe online with gas chromatography-mass spectrometry: Determination of polycyclic aromatic hydrocarbons in water. Journal of Chromatography A 2006; 1114:234-238. http://dx.doi.org/10.1016/j.chroma.2006.02.024

8 Zhao EC, Shan WL, Jiang SR, Liu Y, Zhou ZQ. Determination of the chloroacetanilide herbicides in waters using single-drop microextraction and gas chromatography. Microchemical Journal 2006; 83:105-110. http://dx.doi.org/10.1016/j.microc.2006.03.008

9 Esteve-Turrillas FA, Aman CS, Pastor A, Guardia M. Microwave-assisted extraction of pyrethroid insecticides from soil. Analytical. Chimica Acta 2004; 522:73-78.http://dx.doi.org/10.1016/j.aca.2004.06.039

10 Chong SL, Wang DX, Hayes JD, Wilhite BW, Malik A. Sol-Gel Coating Technology for the Preparation of Solid-Phase Microextraction Fibers of Enhanced Thermal Stability. Analytical Chemistry 1997; 69:3889-3898. http://dx.doi.org/10.1021/ac9703360

11 Arthur CL, Pawliszyn J. Solid phase microextraction with thermal desorption using fused silica optical fibers. Analytical Chemistry 1990; 62:2145-2148. http://dx.doi.org/10.1021/ac00218a019

12 Pawliszyn J, Belardi R. The Application of Chemically Modified Fused Silica Fibres in Extraction of Organics from Water Matrix Samples, and their Rapid Transfer to Capillary Column. Water Pollution Research Journal of Canada 1989; 24:179-191.

13 Helaleh MIH, Fujii S, Korenaga T. Column silylation method for determining endocrine disruptors 
from environmental water samples by solid phase micro-extraction. Talanta 2001; 54:1039-1047. http://dx.doi.org/10.1016/S0039-9140(01)00386-1

Ezquerro Ó, Ortiz G, Pons B, Tena MT. Determination of benzene, toluene, ethylbenzene and xylenes in soils by multiple headspace solid-phase microextraction. Journal of Chromatography A 2004; 1035:17-22. http://dx.doi.org/10.1016/j.chroma.2004.02.030

15 Gorlo D, Wolska L, Zygmunt B, Namiesnik J. Calibration procedure for solid phase microextraction--gas chromatographic analysis of organic vapours in air. Talanta 1997; 44:1543-1550. http://dx.doi.org/10.1016/S0039-9140(96)02176-5

16 Basheer C, Lee HK. Hollow fiber membrane-protected solid-phase microextraction of triazine herbicides in bovine milk and sewage sludge samples. Journal of Chromatography A 2004; 1047:189-194.

17 De Martinis BS, Martins Ruzzene MA, Martin CCS. Determination of ethanol in human blood and urine by automated headspace solid-phase microextraction and capillary gas chromatography. Analytica Chimica Acta 2004; 522:163-168. http://dx.doi.org/10.1016/j.aca.2004.07.007

18 Deng C, Zhang J, Yu X, Zhang W, Zhang X. Determination of acetone in human breath by gas chromatography-mass spectrometry and solid-phase microextraction with on-fiber derivatization. Journal of Chromatography B 2004; 810:269-275.

19 Bermejo AM, López P, Álvarez I, Tabernero MJ, Fernández P. Solid-phase microextraction for the determination of cocaine and cocaethylene in human hair by gas chromatography-mass spectrometry. Forensic Science International 2006;156:2-8. http://dx.doi.org/10.1016/j.forsciint.2005.09.007

20 Plutowska B, Wardencki W. Aromagrams Aromatic profiles in the appreciation of food quality. Food Chemistry 2007; 101:845-872. http://dx.doi.org/10.1016/j.foodchem.2005.12.028

21 Ibáñez E, López-Sebastián S, Ramos E, Tabera J, Reglero G. Analysis of volatile fruit components by headspace solid-phase microextraction. Food Chemistry 1998; 63:281-286. http://dx.doi.org/10.1016/S0308-8146(98)00001-6

22 Azodanlou R, Darbellay C, Luisier JL, Villettaz JC, Amado R. Development of a model for quality assessment of tomatoes and apricots. LebensmittelWissenschaft und-Technologie 2003; 36:223-233.

23 Yu J, Dong L, Wu C, Wu L, Xing J. Hydroxyfullerene as a novel coating for solid-phase microextraction fiber with sol-gel technology. Journal of Chromatography
A 2002; 978:37-48. http://dx.doi.org/10.1016/S00219673(02)01347-X

24 Liu Y, Lee ML, Hageman KJ, Yang Y. Solid-Phase Microextraction of PAHs from Aqueous Samples Using Fibers Coated with HPLC Chemically Bonded Silica Stationary Phases. Analytical Chemistry 1997; 69:5001-5005. http://dx.doi.org/10.1021/ac970686m

25 Kuo C-P, Shiea J. Application of Direct Electrospray Probe To Analyze Biological Compounds and To Couple to Solid-Phase Microextraction To Detect Trace Surfactants in Aqueous Solution. Analytical Chemistry 1999; 71:4413-4417.http://dx.doi.org/10.1021/ac990049r

26 Djozan D, Assadi Y. Monitoring of Polycyclic Aromatic Hydrocarbons in Water Using Headspace Solid-Phase Microextraction and Capillary Gas Chromatography. Microchemical Journal 1999; 63:276-284. http://dx.doi. org/10.1006/mchj.1999.1791

27 Djozan D, Assadi Y, Haddadi SH. Anodized Aluminum Wire as a Solid-Phase Microextraction Fiber. Analytical Chemistry 2001; 73:4054-4058. http://dx.doi.org/10.1021/ac0100188

$28 \mathrm{Wu}$ J, Pawliszyn J. Preparation and applications of polypyrrole films in solid-phase microextraction. Journal of Chromatography A 2001; 909:37-52. http://dx.doi.org/10.1016/S0021-9673(00)01025-6

$29 \mathrm{Wu}$ J, Pawliszyn J. Polypyrrole-Coated Capillary Coupled to HPLC for In-Tube Solid-Phase Microextraction and Analysis of Aromatic Compounds in Aqueous Samples. Analytical Chemistry 2001; 73:55-63. http://dx.doi.org/10.1021/ac000885x

30 Minjia H, Chao T, Qunfang Z, Guibin J. Preparation of polyaniline coating on a stainless-steel wire using electroplating and its application to the determination of six aromatic amines using headspace solid-phase microextraction. Journal of Chromatography A 2004; 1048:257-262.

31 Djozan DB, Bahar S. Solid-Phase Microextraction of Aliphatic Alcohols Based on Polyaniline Coated Fibers. Chromatographia 2004; 59(1-2):95-99.

32 Bagheri H, Mir A, Babanezhad E. An electropolymerized aniline-based fiber coating for solid phase microextraction of phenols from water. Analytica Chimica Acta 2005; 532:89-95. http://dx.doi.org/10.1016/j.aca.2004.10.040

33 Koster EHM, Crescenzi C, Hoedt W den, Ensing K, Jong GJ. Fibers Coated with Molecularly Imprinted Polymers for Solid-Phase Microextraction. Analytical Chemistry 2001; 73:3140-3145. http://dx.doi.org/10.1021/ac001331x

34 Djozan D, Ebrahimi B. Preparation of new solid phase micro extraction fiber on the basis of atrazinemolecular imprinted polymer: Application for GC and 
GC/MS acreening of triazine herbicides in water, rice and onion. Analytica Chimica Acta 2008; 616:152-159. http://dx.doi.org/10.1016/j.aca.2008.04.037

35 Farajzadeh MA, Rahmani NA. Electrolytically produced copper(I) chloride on the copper wire as an excellent sorbent for some amines. Talanta 2005; 65:700-704. http://dx.doi.org/10.1016/j.talanta.2004.07.039

36 Yun L. High extraction efficiency solid-phase microextraction fibers coated with open crown ether stationary phase using sol-gel technique. Analytica Chimica Acta 2003; 486:63-72. http://dx.doi. org/10.1016/S0003-2670(03)00491-4

37 Zeng Z, Qiu W, Huang Z. Solid-Phase Microextraction Using Fused-Silica Fibers Coated with Sol-Gel-Derived Hydroxy-Crown Ether. Analytical Chemistry 2001; 73:2429-2436. http://dx.doi.org/10.1021/ac0012750

38 Kubota LT, Alfaya AAS. A Utilização de materiais obtidos pelo processo de sol-gel na construção de biossensores. Química Nova 2002; 25:835-841.

39 Wang Z, Xiao C, Wu C, Han H. High-performance polyethylene glycol-coated solid-phase microextraction fibers using sol-gel technology. Journal of Chromatography A 2000; 893:157-168. http://dx.doi. org/10.1016/S0021-9673(00)00692-0

40 Kumar A, Gaurav K, Malik A, Tewary DK, Singh B. A review on development of solid phase microextraction fibers by sol-gel methods and their applications. Analytica Chimica Acta 2008; 610:1-14. http://dx.doi.org/10.1016/j.aca.2008.01.028

41 Mohammadi A, Yamini Y, Alizadeh N. Dodecylsulfatedoped polypyrrole film prepared by electrochemical fiber coating technique for headspace solid-phase microextraction of polycyclic aromatic hydrocarbons. Journal of Chromatography A 2005; 1063:1-8. http://dx.doi.org/10.1016/j.chroma.2004.11.087

$42 \mathrm{Wu}$ J, Mullett WM, Pawliszyn J. Electrochemically Controlled Solid-Phase Microextraction Based on Conductive Polypyrrole Films. Analytical Chemistry 2002; 74:4855-4859. http://dx.doi.org/10.1021/ac025595q

43 Alizadeh N, Zarabadipour H, Mohammadi A. Headspace solid-phase microextraction using na electrochemically deposited dodecylsulfate-doped polypyrrole film to determine of phenolic compounds in water. Analytica Chimica Acta 2007; 605:159-165. http://dx.doi.org/10.1016/j.aca.2007.10.039

44 Djozan D, Assadi Y. Monitoring of Phenol and 4-Chlorophenol in Petrochemical Sewage Using Solid-Phase Microextraction and Capillary Gas Chromatography. Chromatographia 2003; 58:637-642.
45 Sun T, Jia J, Fang N, Wang Y. Application of novel activated carbon fiber solid-phase, microextraction to the analysis of chlorinated hydrocarbons in water by gas chromatography-mass spectrometry. Analytica Chimica Acta 1997; 530:33-40. http://dx.doi.org/10.1016/j.aca.2004.08.042

46 Liu Y, Shen Y, Lee ML. Porous Layer Solid Phase Microextraction Using Silica Bonded Phases. Analytical Chemistry 2005; 69:190-195. http://dx.doi.org/10.1021/ac960791g

47 Hou J-G, Ma Q, Du X-Z, Deng H-I, Gao J-Z. Inorganic/ organic mesoporous silica as a novel fiber coating of solid-phase microextraction. Talanta 2004; 62:241-246. http://dx.doi.org/10.1016/j.talanta.2003.07.003

48 Li X, Li C, Chen J, Li C, Sun C. Polythiophene as a novel fiber coating for solid-phase microextraction. Journal of Chromatography A 2008; 1198:7-13. http://dx.doi.org/10.1016/j.chroma.2008.05.051

49 Cao D-D, Lu J-X, Liu J-F, Jiang G-B. In situ fabrication of nanostructured titania coating on the surface of titanium wire: A new approach for preparation of solid-phase microextraction fiber. Analytica Chimica Acta 2008; 611:56-61. http://dx.doi.org/10.1016/j.aca.2008.01.067

50 Setkova L, Risticevic S, Pawliszyn J. Rapid headspace solid-phase microextraction-gas chromatographictime-of-flight mass spectrometric method for qualitative profiling of ice wine volatile fraction - I: Method development and optimization. Journal of Chromatography A 2007; 1147:213-223. http://dx.doi.org/10.1016/j.chroma.2007.02.058

51 Setkova L., Risticevic S., Pawliszyn J. Rapid headspace solid-phase microextraction-gas chromatographictime-of-flight mass spectrometric method for qualitative profiling of ice wine volatile fraction II: Classification of Canadian and Czech ice wines using statistical evaluation of the data. Journal of Chromatography A 2007; 1147:224-240. http://dx.doi.org/10.1016/j.chroma.2007.02.052

52 Setkova L, Risticevic S, Linton CM, Ouyang G, Bragg LM. Pawliszyn J. Solid-phase microextraction-gas chromatography-time-of-flight mass spectrometry utilized for the evaluation of the new-generation super elastic fiber assemblies. Analytica Chimica Acta 2007; 581:221-231. http://dx.doi.org/10.1016/j.aca.2006.08.022

53 Oliveira AF, Silveira CB, Campos SD, Campos EA, Carasek E. The Use of a Thin Glass-Ceramic Rod as a Surface for Sol-Gel Coating in the Preparation of SPME Fibers. Chromatographia 2005; 61:277-283. http://dx.doi.org/10.1365/s10337-005-0507-x 
54 Zhou F, Li X, Zeng Z. Determination of phenolic compounds in wastewater samples using a novel fiber by solid-phase microextraction coupled to gas chromatography. Analytica Chimica Acta 2005; 583:63-70.http://dx.doi.org/10.1016/j.aca.2005.02.009

$55 \mathrm{Yu}$ J, Wu C, Xing J. Development of new solid-phase microextraction fibers by sol-gel technology for the determination of organophosphorus pesticide multiresidues in food. Journal of Chromatography A 2004; 1036:101-111. http://dx.doi.org/10.1016/j. chroma.2004.02.081

56 Lopes AL, Augusto F. Preparation and characterization of polydimethylsiloxane/poly(vinylalcohol) coated solid phase microextraction fibers using sol-gel technology. Journal of Chromatography A 2004; 1056:13-19. http://dx.doi.org/10.1016/j.chroma.2004.05.047

57 Cai L, Xing J, Dong L, Wu C. Application of polyphenylmethylsiloxane coated fiber for solid-phase microextraction combined with microwave-assisted extraction for the determination of organochlorine pesticides in Chinese teas. Journal of Chromatography A 2003; 1015:11-21. http://dx.doi.org/10.1016/S00219673(03)01328-1

58 Liu M, Zeng Z, Xiong B. Preparation of novel solidphase microextraction fibers by sol-gel technology for headspace solid-phase microextraction-gas chromatographic analysis of aroma compounds in beer. Journal of Chromatography A 2005; 1065:287-299. http://dx.doi.org/10.1016/j.chroma.2004.12.073

$59 \mathrm{Hu}$ Y-I, Fu Y-I, Li G-K. Preparation of anilinemethyl triethoxy silane/polydimethylsiloxane sol-gel coatings for solid-phase microextraction of aromatic compounds. Analytica Chimica Acta2006;567:211-217. http://dx.doi.org/10.1016/j.aca.2006.03.042

60 Liu M, Zeng Z, Tian Y. Elimination of matrix effects for headspace solid-phase microextraction of important volatile compounds in red wine using a novel coating. Analytica Chimica Acta 2005; 540:341-353. http://dx.doi.org/10.1016/j.aca.2005.03.029

61 Bagheri H, Babanezhad E, Khalilian F. A novel sol-gel-based amino-functionalized fiber for headspace soli-phase microextraction of phenol and chlorophenols from environmental samples. Analytica Chimica Acta 2008; 616:49-55. http://dx.doi.org/10.1016/j.aca.2008.04.008
62 Zeng J, Chen J, Lin Z, Chen W, Chen X, Wang X. Development of polymethylphenylsiloxane-coated fiber for solid-phase microextraction and ist analytical application of qualitative and semi-quantitative of organochlorine and pyrethroid pesticides in vegetables. Analytica Chimica Acta 2008; 619:59-66. http://dx.doi.org/10.1016/j.aca.2008.02.013

63 Zhou J, Yang F, Cha D, Zeng Z, Xu Y. Headspace solid-phase microextraction with novel sol-gel permethylated-b-cyclodextrin/hydroxyl-termination silicone oil fiber for determination of polybrominated diphenyl ethers by gas chromatography-mass spectrometry in soil. Talanta 2007; 73:870-877. http://dx.doi.org/10.1016/j.talanta.2007.05.006

64 Farhadi K, Mamaghanian M, Maleki RA. A sol-gel based solid phase microextraction fiber for analysis of aromatic hydrocarbons. Journal of Hazardous Materials 2008; 152:677-682. http://dx.doi.org/10.1016/j.jhazmat.2007.07.057

65 Tan L, Crone WC. Surface characterization of NiTi modified by plasma source ion implantation. Acta Materialia 2002; 50:4449-4460. http://dx.doi. org/10.1016/j.jhazmat.2007.07.057

66 Budziak D, Mardendal E, Carasek E. Preparation and characterization of new solid-phase microextraction fibers obtained by sol-gel technology and zirconium oxide electrodeposited on NiTialloy. Journal of Chromatography A 2008; 1187(1-2):34-39. PMid:18314128. http://dx.doi. org/10.1016/j.chroma.2008.02.003

67 Budziak D, Mardendal E, Carasek E. Application of poly(dimethylsiloxane) fiber sol - Gel coated onto NiTi alloy electrodeposited with zirconium oxide for the determination of organochlorine pesticides in herbal infusions. Journal of Separation Sience 2008; 31:2875-2881. http://dx.doi.org/10.1002/jssc.200800130

68 Budziak D, Mardendal E, CarasekE. New poly(ethylene glycol) solid-phase microextraction fiber employing zirconium oxide electrolytically deposited onto a NiTi alloy as substrate for sol-gel reactions. Journal of Chromatography A 2008; 1158:54-58. http://dx.doi. org/10.1016/j.chroma.2008.05.048

69 Budziak D, Mardendal E, Carasek E. Application of robust $\mathrm{NiTi}-\mathrm{ZrO}_{2}$-PEG SPME fiber in the determination of haloanisoles in cork stopper samples. Analytica Chimica Acta 2008; 629:92-98. http://dx.doi.org/10.1016/j.aca.2008.09.038 